\title{
The Lipid Moiety of Haemozoin (Malaria Pigment) and $P$. falciparum Parasitised Red Blood Cells Bind Synthetic and Native Endothelin-1
}

\author{
Nicoletta Basilico, ${ }^{1}$ Silvia Parapini, ${ }^{1}$ Francesca Sisto, ${ }^{1}$ Fausta Omodeo-Salè, ${ }^{2}$ \\ Paolo Coghi, ${ }^{3}$ Fernando Ravagnani, ${ }^{4}$ Piero Olliaro, ${ }^{5}$ and Donatella Taramelli ${ }^{1}$ \\ ${ }^{1}$ Dipartimento di Sanità Pubblica-Microbiologia-Virologia, Università degli Studi di Milano, via Pascal 36, 20133 Milan, Italy \\ ${ }^{2}$ Dipartimento di Scienze Molecolari Applicate ai Biosistemi (DISMAB), Università degli Studi di Milano, \\ via Trentacoste 2, 20134 Milan, Italy \\ ${ }^{3}$ ISTM, CNR, via Venezian 21, 20133 Milan, Italy \\ ${ }^{4}$ Fondazione IRCCS, Istituto Nazionale dei Tumori, via Venezian 1, 20133 Milan, Italy \\ ${ }^{5}$ UNICEF/UNDP/WB/WHO Special Programme for Research \& Training in Tropical Diseases (TDR), Avenue Appia 20, \\ 1211 Geneva 27, Switzerland
}

Correspondence should be addressed to Nicoletta Basilico, nicoletta.basilico@unimi.it

Received 3 August 2009; Revised 8 November 2009; Accepted 29 December 2009

Academic Editor: Abhay R. Satoskar

Copyright ( $) 2010$ Nicoletta Basilico et al. This is an open access article distributed under the Creative Commons Attribution License, which permits unrestricted use, distribution, and reproduction in any medium, provided the original work is properly cited.

Endothelin1 (ET-1) is a 21-amino acid peptide produced by the vascular endothelium under hypoxia, that acts locally as regulator of vascular tone and inflammation. The role of ET-1 in Plasmodium falciparum malaria is unknown, although tissue hypoxia is frequent as a result of the cytoadherence of parasitized red blood cell (pRBC) to the microvasculature. Here, we show that both synthetic and endothelial-derived ET-1 are removed by parasitized RBC (D10 and W2 strains, chloroquine sensitive, and resistant, resp.) and native haemozoin (HZ, malaria pigment), but not by normal RBC, delipidized HZ, or synthetic beta-haematin (BH). The effect is dose dependent, selective for ET-1, but not for its precursor, big ET-1, and not due to the proteolysis of ET1. The results indicate that ET-1 binds to the lipids moiety of HZ and membranes of infected RBCs. These findings may help understanding the consequences of parasite sequestration in severe malaria.

\section{Introduction}

Plasmodium falciparum (P.f.) infection may evolve into severe disease if untreated or inadequately treated, causing an estimated one million deaths annually [1]. The severe forms of malaria are characterized by the systemic release of inflammatory cytokines and the cytoadherence of P.f. parasitized red blood cells (pRBCs) to the vascular endothelium. This results in the sequestration of pRBCs in various organs, such as the brain, lung, and placenta, causing microcirculatory obstruction and subsequent tissue hypoxia, metabolic disturbances, and multiorgan failure [2-4].

Products of parasite origin, such as haemozoin (HZ, malaria pigment), contribute to the pathogenesis of severe malaria by increasing the production of host inflammatory cytokines and the expression of adhesion molecules on the endothelium [4-9]. HZ, the detoxification product of haem, accumulates as insoluble crystals inside the food vacuole of intraerythrocytic parasites and, once released into the circulation, is phagocytised by host cells. Both native $\mathrm{HZ}$ and its synthetic analogue, $\beta$-haematin $(\mathrm{BH})$, can modulate phagocyte and endothelial cell functions in vitro [10-12].

The sequestration of $\mathrm{pRBC}$ in the microvasculature may reduce local oxygen tension with increased risk of tissue hypoxia, hence inducing the transcriptionally regulated expression of vasoactive substances in order to modulate the vascular tone. One of these products is endothelin-1 (ET-1), a potent vasoconstrictor agent, which is produced 
by the vascular endothelium in response to various stimuli including hypoxia and cytokines $[13,14]$. ET- 1 is a 21 -amino acid long peptide derived from a larger precursor, pre-proET1 that is cleaved first to big ET-1 (whose vasoconstrictor activity is approximately two orders of magnitude lower than that of ET-1 itself) and then converted to ET-1 by neutral metallo-endopeptidases known as endothelinconverting enzymes (ECEs). ET-1 exerts various important biological actions mediated by three receptor subtypes, ET$\mathrm{A}, \mathrm{ET}-\mathrm{B}$, and ET-C, which have been identified in various human tissues [15].

The expression and/or production of endothelins and their receptors is increased in several pathological conditions such as cardiovascular diseases, pulmonary hypertension, renal failure, and subarachnoid haemorrhage [16-18]. Elevated levels of ET-1 are also associated with serious infectious diseases, such as bacterial sepsis and trypanosomiasis [1921].

The role of ET-1 in falciparum malaria is still controversial and largely unknown. It has been reported that severe malaria patients have increased plasma levels of big ET-1 which correlate with the levels of TNF- $\alpha$, but not with parasitaemia, fever, or other features of severe infection [22]. Recently, children with malaria were shown to have significantly higher plasma levels of ET-1 compared to healthy controls. However, there was an apparent trend for those with severe malaria to have lower ET-1 levels compared to uncomplicated malaria patients, although a direct relationship with parasitaemia was not evident [23]. In the previous in vitro studies, we observed that pRBC down modulate the constitutive or hypoxia-induced ET-1 production by microvascular endothelial cells indicating that pRBC may induce local modifications of the vascular tone and the inflammatory response $[24,25]$. To obtain further insights on the relevance of ET-1 modulation for severe malaria, we performed the experiments herewith presented to identify the parasite product(s) and the mechanism through which $\mathrm{pRBC}$ modulate the production and/or the activity of ET-1 under normoxic or hypoxic conditions.

\section{Materials and Methods}

2.1. Cells, Reagents, and Plasmodium falciparum Cultures. A long-term cell line of dermal microvascular endothelial cells (HMECs-1) immortalized by SV 40 large $\mathrm{T}$ antigen was kindly provided by Dr. F. Candal, the Center for Disease Control, Atlanta, GA, USA [26]. Cells were maintained in MCDB 131 medium (Invitrogen, Milan, Italy) supplemented with $10 \%$ fetal calf serum (HyClone), $10 \mathrm{ng} / \mathrm{mL}$ of epidermal growth factor (Chemicon), $1 \mu \mathrm{g} / \mathrm{mL}$ of hydrocortisone, $2 \mathrm{mM}$ glutamine, 100 units $/ \mathrm{mL}$ of penicillin, $100 \mu \mathrm{g} / \mathrm{mL}$ of streptomycin, and $20 \mathrm{mM}$ Hepes buffer, pH 7.4 (EuroClone). Unless otherwise stated, all reagents were from Sigma Aldrich, Milan, Italy.

In vitro cultures of P.f. were adapted from Trager and Jensen [27]. A chloroquine (CQ)-sensitive P.f. strain (D10) and a CQ-resistant strain (W2) were maintained at $5 \%$ haematocrit (human type $\mathrm{A} \mathrm{RBC}$ ) at $37^{\circ} \mathrm{C}$ in $\mathrm{RPMI}$
1640 medium (GIBCO BRL) supplemented with 10\% heatinactivated $\mathrm{A}+$ human plasma, $20 \mathrm{mM}$ Hepes buffer $\mathrm{pH} 7.4$ in a standard gas mixture consisting of $1 \% \mathrm{O}_{2}, 5 \% \mathrm{CO}_{2}, 94 \%$ $\mathrm{N}_{2}$.

2.1.1. Preparation of $H Z$ (Native and Delipidized) and $\beta$ Haematin (BH). To isolate HZ, pRBC (4-8\% parasitaemia) were washed twice with serum-free culture medium, resuspended to $25 \%$ haematocrit, and fractionated on a discontinuous Percoll $/ 4 \%$ sorbitol (wt/vol) gradient $(0,40,80 \%)$ [28]. After centrifugation at $1075 \mathrm{~g}, \mathrm{HZ}$ was collected at the top of the gradient, $0-40 \%$ interphase, washed three times with PBS, and stored at $-20^{\circ} \mathrm{C}$.

For the preparation of the delipidized $\mathrm{HZ}$, native $\mathrm{HZ}$ was washed 3 times with Tris- $\mathrm{HCl}$ buffer $(10 \mathrm{mM}, \mathrm{pH}$ 7.5) and the pellet extracted twice in a mixture of chloroform/methanol $2: 1(\mathrm{v} / \mathrm{v})$ and twice in chloroform/methanol $1: 2(\mathrm{v} / \mathrm{v})$. At the end, the pellet, containing the delipidized $\mathrm{HZ}$ was dried under a flux of nitrogen and resuspended in Tris- $\mathrm{HCl}$ buffer.

Synthetic malaria pigment, $\beta$-haematin (BH), was synthesized from haematin (Sigma, Milan, Italy) in methanol as described [29]. The haem content of a weighed amount of the native or delipidized $\mathrm{HZ}$ and $\mathrm{BH}$ dissolved in $0.1 \mathrm{M}$ $\mathrm{NaOH}$ was determined by reading the absorbance at $385 \mathrm{~nm}$ $\left(\varepsilon_{385}\right.$ hematin $\left.=6.1 \times 10^{4} \mathrm{M} \mathrm{cm}^{-1}\right)($ Soret band $)$.

2.2. HMEC-1 Treatment. HMECs-1 were seeded in quadruplicate at $1.5 \times 10^{4} /$ well in 96 well flat bottom tissue culture clusters (Costar, 3596). After overnight incubation, monolayers were exposed to asynchronous P.f. cultures. pRBCs were added to the cells at $1 \%$ haematocrit with a parasitaemia ranging between 2 and 4\%. Uninfected RBCs were used as controls in all experiments. In some experiments, HMECs-1 were exposed to HZ isolated from the $\mathrm{pRBC}$ cultures as described above.

HMECs- 1 treated with pRBC or haemozoin were cultured for 24 hours at $37^{\circ} \mathrm{C}$ under normoxic $\left(20 \% \mathrm{O}_{2}, 5 \%\right.$ $\left.\mathrm{CO}_{2}, 75 \% \mathrm{~N}_{2}\right)$ or hypoxic conditions $\left(1 \% \mathrm{O}_{2}, 5 \% \mathrm{CO}_{2}, 94 \%\right.$ $\mathrm{N}_{2}$ ) in sealed chambers.

2.3. Quantification of ET-1 and Big ET-1. At the end of each treatment, plates were centrifuged and ET-1 and big ET-1 peptides quantified in the supernatant using a specific ELISA test (PANTEC, Torino, Italy). ET-1 was also determined by a fluorimetric procedure according to Chaloin [30]. Fluorescence spectra were recorded in quarz cuvettes by a Perkin Elmer LS50 spectrofluorimeter at an excitation wavelength of $280 \mathrm{~nm}$ and the area of the peaks were calculated by the FLWinLab software. To correct for the interference on the intensity of the fluorescence caused by the release of small amounts of haemoglobin during incubation, control samples were prepared by diluting ET-1 in the supernatant recovered by centrifugation of a suspension of normal RBC in PBS.

2.4. RT-PCR Detection of ET-1 mRNA. For mRNA and RTPCR analysis, HMEC- 1 were seeded at $5 \times 10^{5} /$ well in 6 well 
plates (Costar, 3516), treated with pRBC or control RBC, and incubated for 24 hours in normoxic or hypoxic conditions. At the end of the treatment cells were lysed in $1 \mathrm{~mL}$ Trizol Reagent (Invitrogen). Total RNA was isolated according to the manufacturer instructions. $1 \mu \mathrm{g}$ of total RNA was reversetranscribed using Superscript First-Strand Synthesis System (Invitrogen, USA), according to the manufacturer's protocol. A portion of cDNA $(0.2 \mu \mathrm{g})$ was then used as the template in subsequent PCR amplifications in a total volume of $30 \mu \mathrm{L}$ containing $1 \mathrm{X}$ buffer (Promega), $2.0 \mathrm{mM} \mathrm{MgCl}_{2}$ (Promega), $0.2 \mathrm{mM}$ each dNTP, sense and antisense primers $(0.2 \mu \mathrm{M})$, and 1.25 Units of Taq thermostable polymerase (Promega). The sequences of ET-1 specific primers were: forward $5^{\prime}$ TGC TCC TGC TCG TCC CTG ATG GAT AAA GAG-3', reverse $5^{\prime}$-GGT CAC ATA ACG CTC TCT GGA GGG CTT3' (462-bp fragment). G3PDH control primers were: forward 5'-TGA AGG TCG GAG TCA ACG GAT TTG GT-3', reverse 5'-CAT GTG GGC CAT GAG GTC CAC-3' CAC (983-bp fragment). Amplification was performed for 30 cycles and the annealing temperature was $58^{\circ} \mathrm{C}$. A negative control was included in each assay to confirm that only cDNA PCR products were detected and that none of the reagents was contaminated with cDNA of previous PCR products.

All PCR products were analyzed by electrophoresis on $1 \%$ agarose gels and visualized by ethidium bromide staining.

2.5. Incubation of ET-1 and Big ET-1 Peptides with RBC of P.f. Cultures. Different concentrations of ET-1 or big ET-1 $(5.0,2.5,1.2 \mathrm{fmol} / \mathrm{mL}$ ) (Calbiochem Corp.) were added to pRBC suspension (2-5\% parasitaemia, $2 \%$ haematocrit) in $100 \mu \mathrm{l}$ of HMEC-1 culture medium distributed in 96 well flat bottom microplates. Control unparasitized RBCs were maintained for 3-4 days before the experiment in the same conditions of pRBC $\left(5 \%\right.$ haematocrit at $\left.37^{\circ} \mathrm{C}\right)$ and incubated with ET-1 or big ET-1 as described. After 2, 4, 6, 24 hours of incubation, plates were centrifuged and supernatants were collected and assayed for the presence of ET-1 or big ET-1. ET-1 $(5 \mathrm{fmol} / \mathrm{mL})$ was incubated for 24 hours with pRBC in the presence of the receptor ET-A or ET-B antagonists, alone or combined, (0.5-0.1 $\mu \mathrm{M})$ (JKC302 and BQ788, resp.) (Sigma) or a Protease Inhibitor Cocktail (used at a dilution of 1 : 200) (P1860 Sigma). ET-1 was then assayed in the supernatants.

Since parasite cultures are a heterogeneous population of cells made of pRBC and normal RBC of different density, the total RBC suspension was fractionated onto a Percoll/4\% sorbitol (wt/vol) gradient [28]. This procedure allows the separation of the HZ containing fraction (top of the gradient), $\mathrm{pRBC}$ (density $=1.078$ ), and three fractions (young, medium, and old) of unparasitized RBC. Each fraction was incubated with ET-1 $(5-2.5 \mathrm{fmol} / \mathrm{mL})$ for 24 hours after which the residual ET-1 was determined in the supernatant. Delipidized HZ, BH, or the supernatants of P.f. cultures were also employed.

When ET-1 was determined by fluorescence spectroscopy, normal uninfected RBC, pRBC (D10 strain, 2\% haematocrit) were incubated in PBS in the presence of ET-1 $(10 \mathrm{pmol} / \mathrm{mL})$ for 18 hours, in $2 \mathrm{~mL}$ final volume.
Samples were then centrifuged at $600 \times \mathrm{g}$ for 10 minutes and supernatants recovered for the fluorimetric analysis.

2.6. Statistical Analysis. All the assays were performed at least three times in quadruplicate and the results analyzed by the Student's paired $t$-test. Statistical significance was set at $P<$ .05 .

\section{Results}

3.1. Impaired Recovery of ET-1 Produced by HMEC-1 Cocultured with P.f. $p R B C$. The constitutive production of ET1 by endothelial HMEC- 1 cells is significantly increased by hypoxia. Coincubation of endothelial cells with pRBC (D10 and W2 strains at $2-4 \%$ parasitaemia), but not with normal uninfected RBC, induced a decrease in the levels of ET1 detected in the supernatants; no differences were seen between the two strains used (Figure 1(a)). The decrease in ET-1 induced by D10 was dose-dependent (see Figure 1(b) and previous work [24]) and was not due to inhibition of ET-1 gene transcription or translation by pRBC since the level of ET-1 mRNA as well as that of big ET-1, the precursor of the active peptide ET-1, was not affected in either normoxic or hypoxic conditions (Figures 1(b) and $1(c))$. We also excluded that pRBC may inhibit the processing of big ET-1 to ET-1 since no accumulation of big ET1 was seen in the supernatants of endothelial cells incubated with $\mathrm{pRBC}$.

3.2. Effect of pRBC on Synthetic ET-1 and Big ET-1. Having determined that $\mathrm{pRBC}$ did not interfere with the production of ET-1 by HMEC-1, we investigated whether pRBCs were able to bind or degrade synthetic ET-1. The first set of experiments was conducted by incubating different concentrations of commercially available, synthetic ET-1 peptide with pRBC for 24 hours and measuring the amount of residual ET-1 in the culture medium by ELISA. In the presence of pRBC (D10 strain, $2-4 \%$ parasitaemia), the levels of ET- 1 in the extracellular medium were significantly reduced while big ET-1, used as control, was not affected (Figure 2(a)). Similar results were obtained with $\mathrm{pRBC}$ from the CQ resistant P.f. strain W2 (data not shown).

The removal of ET-1 by pRBC was a slow process, with a significant reduction of ET-1 (60-70\% compared to control) occurring only after 24 hours (Figure 2(b)). ET1 levels were not reduced following incubation of ET-1 with uninfected RBC (Figure 2(b)). The decrease of ET-1 in the presence of $\mathrm{pRBC}$ was confirmed using a fluorimetric method of quantification of ET-1; the data of a representative experiment are reported in Table 1. A good correlation $\left(r^{2}=0.939\right)$ between the \% of ET-1 recovery obtained by the two methods was achieved. The reduction of ET-1 after incubation with pRBC was not due to the enzymatic degradation of the peptide or to soluble factors released by $\mathrm{pRBC}$ as shown by the experiments conducted in the presence of protease inhibitors or supernatants from cultures of different P.f. strains (Table 2). This suggests that ET-1 was not degraded, but likely bound to pRBC membrane. 
TABLE 1: Absorption of ET-1 by pRBC (D10 strain) or haemozoin: comparison between fluorimetric detection and ELISA assay.

\begin{tabular}{|c|c|c|c|c|}
\hline \multirow[b]{3}{*}{ Samples } & \multicolumn{4}{|c|}{ ET-1 recovery } \\
\hline & \multicolumn{2}{|c|}{ Fluorescence spectroscopy } & \multicolumn{2}{|c|}{ ELISA } \\
\hline & Fluorescence Intensity (AUC) & $\%$ control & $\mathrm{pmol} / \mathrm{mL}$ & $\%$ control \\
\hline Control $^{\mathrm{a}}$ & 41305 & & 9.04 & \\
\hline Normal RBC & 40503 & 98.1 & 8.69 & 96.1 \\
\hline pRBC (D10 1.6\%) & 32224 & 78.0 & 5.93 & 65.5 \\
\hline pRBC (D10 3.2\%) & 30784 & 74.5 & 5.03 & 55.6 \\
\hline
\end{tabular}

Data of a representative experiment out of three experiments performed in the same conditions. ET-1 ( 10 pmol/mL) was incubated for 18 hours in PBS or in the presence of normal RBC or pRBC (D10 strain at 1.6\% and 3.2\% parasitaemia). ${ }^{a}$ Control samples for fluorescence spectroscopy were prepared by diluting ET-1 in the supernatant recovered by centrifugation of a suspension of normal RBC incubated in PBS for the same length of time. Control samples for ELISA test were prepared by diluting ET-1 in PBS. At the end of the incubation, RBCs were centrifuged and supernatants used for ET-1 determination by both methods.

Data are expressed as arbitrary fluorescent units (AUC Area Under the Curve) or as pmol/mL.

TABLE 2: Effect of normal RBC, pRBC, or supernatants from $P$. falciparum cultures on the recovery of synthetic ET-1.

\begin{tabular}{|c|c|c|c|c|c|c|c|}
\hline \multicolumn{8}{|c|}{ \% ET-1 recovery when incubated with: } \\
\hline & Medium & Normal RBC & $\mathrm{pRBC}(\mathrm{D} 10)$ & $\mathrm{pRBC}(\mathrm{W} 2)$ & SN normal RBC & SN pRBC (D10) & SN pRBC (W2) \\
\hline ET-1 $1^{a} 5$ & 100 & $91.96 \pm 7.2$ & $39.8 \pm 8.3^{*}$ & $40.2 \pm 7.5^{*}$ & $102 \pm 5.4$ & $98 \pm 6.3$ & $96 \pm 7.8$ \\
\hline+ proteaseinhibitor & $105.7 \pm 2.1$ & $101.7 \pm 4.8$ & $29.6 \pm 7.3^{*}$ & $48.3 \pm 11^{*}$ & ND & ND & ND \\
\hline
\end{tabular}

${ }^{a}$ ET-1 $(5 \mathrm{fmol} / \mathrm{mL})$ was incubated for 24 hours in medium or in the presence of normal unparasitized RBC, pRBC from different $P$. falciparum strains or supernatants (SNs) from parasite cultures. ET-1 was also incubated with RBC or pRBC in the presence of a protease inhibitor cocktail. At the end of the incubation, ET-1 was detected by ELISA.

Data are expressed as $\%$ of ET-1 recovery respect to controls and represent the mean \pm SD from three different experiments. ${ }^{*} P<.01$ versus control.

To investigate whether ET-1 receptors were involved in the removal/binding of ET-1 by pRBC, the experiments were repeated using two receptor antagonists, JKC 302 or BQ788 which are specific for the ET-A or ET-B receptors, respectively. As shown in Figure 3, none of the receptor antagonists (used alone or combined) prevented the removal of ET-1 by pRBC suggesting that most of the binding was aspecific. Similar findings were obtained in binding experiments with radio-labelled ET-1 ( $\left.{ }^{125} \mathrm{I}-\mathrm{ET}-1\right)$ (data not shown).

Since parasite cultures are composed by $\mathrm{pRBC}$, normal $\mathrm{RBC}$ of different age/density, parasite debris and free HZ, a Percoll fractionation of the culture was performed, as previously described [28]. Five different fractions were recovered: young, medium and old normal RBC, enriched pRBC (mainly at the trophozoite stage) and free HZ. Each fraction was then incubated with $2.5 \mathrm{fmol} / \mathrm{mL}$ of ET- 1 for 24 hours. As shown in Figure 4, only the pRBC fraction and $\mathrm{HZ}(2 \mu \mathrm{g} / \mathrm{mL})$ significantly bound ET- 1 . This demonstrates that the decrease in the content of ET-1 in HMEC-1 cultures treated with pRBC (Figure 1) was due to binding of the released peptide to $\mathrm{pRBC}$ and/or $\mathrm{HZ}$.

Native HZ purified from parasite cultures is mainly made of crystals of dimers of $\mathrm{Fe}(\mathrm{III})$ protoporphyrin IX (haematin), but includes also proteins and lipids of parasite origin $[31,32]$. Since data in the literature suggest that ET-1 interacts by electrostatic forces with neutral phospholipids, we investigated the role of the lipid fraction in ET-1 binding. To this purpose, the lipid fraction was removed from native $\mathrm{HZ}$ and the delipidized $\mathrm{HZ}$ assayed in the ET-1 adsorption assay. The haem containing portion of $\mathrm{HZ}$ (beta haematin, $\mathrm{BH}$ ) was used as control. The experiment was performed by incubating ET-1 ( $5 \mathrm{fmol} / \mathrm{mL}$ ) with different concentrations of native $\mathrm{HZ}$ or delipidized $\mathrm{HZ}$ or $\mathrm{BH}$. As shown in Figure 5(a) native HZ removed ET-1 in a dose-dependent manner, while delipidized HZ or BH had no effect at any of the doses employed, indicating that the lipid portion, but not the haem moiety of HZ was involved in ET-1 binding.

To verify whether HZ could bind ET-1 produced by endothelial cells, HMECs-1 were treated for 24 hours with different concentrations of native HZ. Similarly to pRBC, HZ reduced the ET- 1 levels in a dose-dependent manner both in normoxic and hypoxic conditions (Figure 5(b)). At the highest dose use $(50 \mu \mathrm{g} / \mathrm{mL})$, the decrease of ET-1 by HZ was $87.1 \%$ and $83.6 \%$ in normoxic or hypoxic conditions, respectively. As expected, BH did not interfere with ET-1 production by HMEC-1 or recovery.

\section{Discussion}

The present study provides an evidence that the decrease in ET-1 caused by P. falciparum-parasitized RBCs is due by the aspecific binding of ET-1 to pRBCs. The reduction of ET-1 levels is specific for ET-1 (its precursor, big ET-1 is unaffected), is not caused by proteolytic degradation by soluble products or ET-1 binding to ET-A or B receptors $[33,34]$, or inhibition of endothelial cells functions by pRBCs [24]. The slow reaction kinetics (18-24 hours of incubation needed for a significant effect to occur) further support this hypothesis. The aspecific binding of ET-1 to pRBC involves 


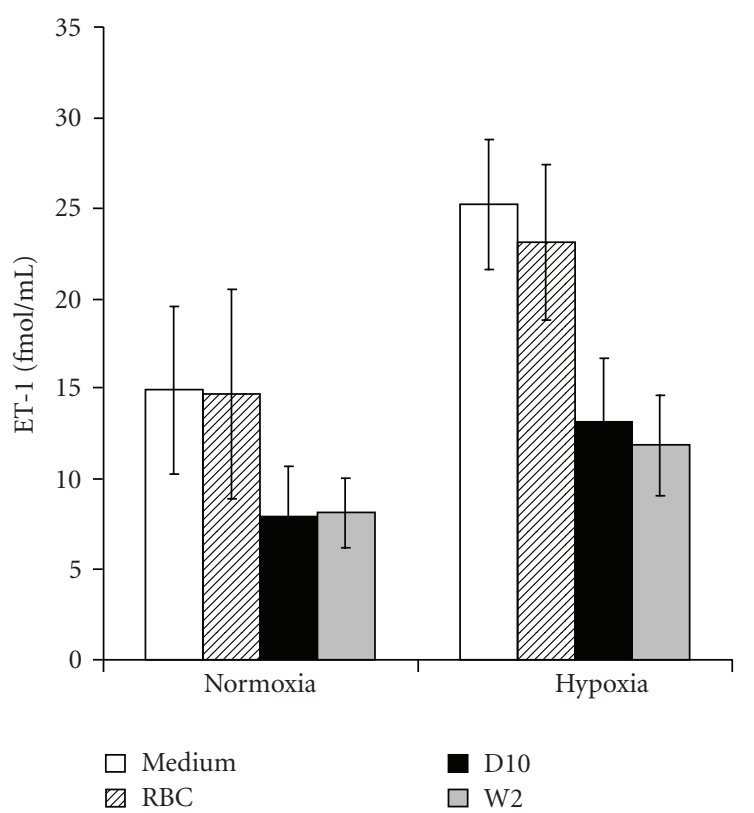

(a)

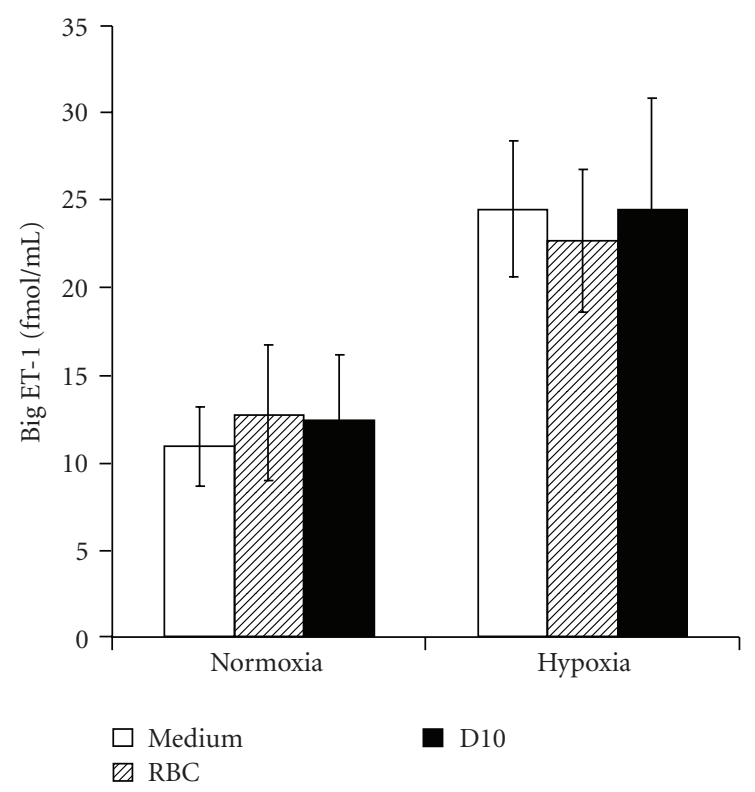

(c)

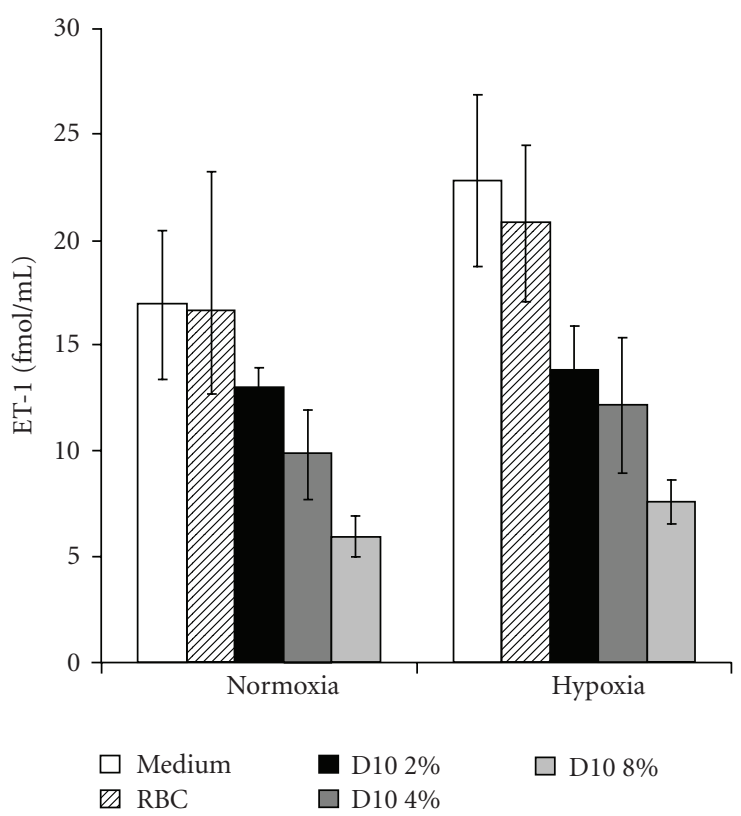

(b)
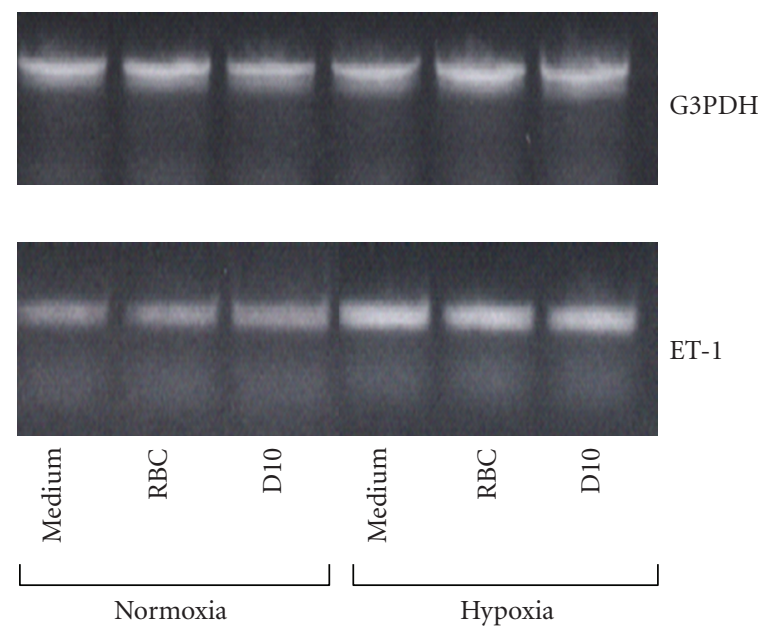

(d)

Figure 1: Effect of pRBC on the release of ET-1 and big ET-1 from human microvascular endothelial cells (HMECs-1). HMECs-1 were treated for 24 hours in the presence of normal RBC or pRBC (D10 or W2 strains) at $2-4 \%$ parasitaemia in (a), (c), (d) or at the indicated levels of parisitaemia (b) under normoxic or hypoxic conditions. Supernatants were then assayed for the presence of ET-1 (a, b) or big ET-1 (c) by ELISA. Results represent the mean \pm SD from five different experiments. (a) $P=.0014$ and $P=.0041$ D10 versus control in normoxia and hypoxia, respectively; $P=.0017$ and $P=.0052 \mathrm{~W} 2$ versus control in normoxia and hypoxia, respectively; (b) $P=.014$ and $P=.033$ : D10 8\% and 4\% versus control, in normoxia; $P=.006, P=.01, P=.027: \mathrm{D} 10$ 8\%, 4\%, and 2\% versus control in hypoxia. (d) RT-PCR analysis of ET-1. G 3 PDH served as control. The cells were lysed and mRNA extracted. The PCR products were separated through agarose gel electrophoresis and visualized by ethidium bromide. The reported data are representative of three independent experiments. 

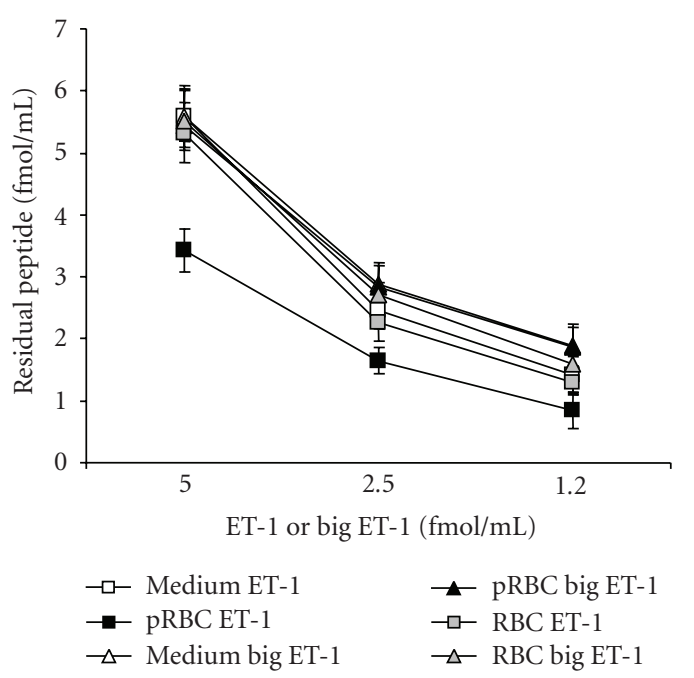

(a)

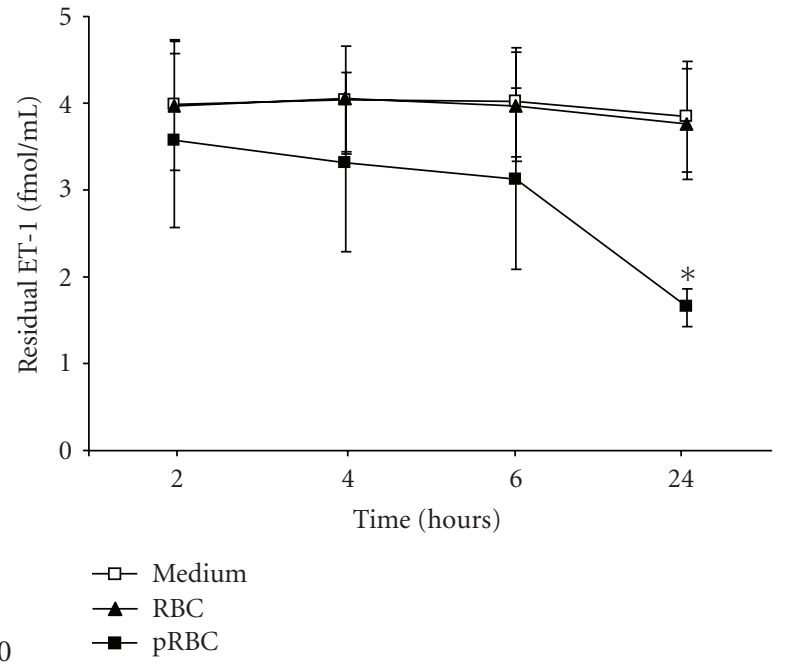

(b)

Figure 2: Effect of incubation of pRBC (D10 strain) or normal RBC on synthetic ET-1 and big Et-1. (a) Different concentrations of ET-1 (square) or big ET-1 (triangle) were incubated in medium (empty symbols) or in the presence of uninfected RBC (grey symbols) or pRBC (black symbols). After 24 hours of incubation, samples were collected and assayed for the presence of the residual ET-1 or big et-1 by ELISA. The results represent the mean \pm SD from three different experiments. (b) ET-1 ( $5 \mathrm{fmol} / \mathrm{mL}$ ) was incubated in medium (empty square) or in the presence of pRBC (black square) or control RBC (black triangle) for 2, 4, 6, 24 hours. At the end of incubation, samples were collected and assayed for the presence of the residual ET- 1 . Results represent mean \pm SD from three different experiments $\left({ }^{*} P=.029\right.$ versus medium).

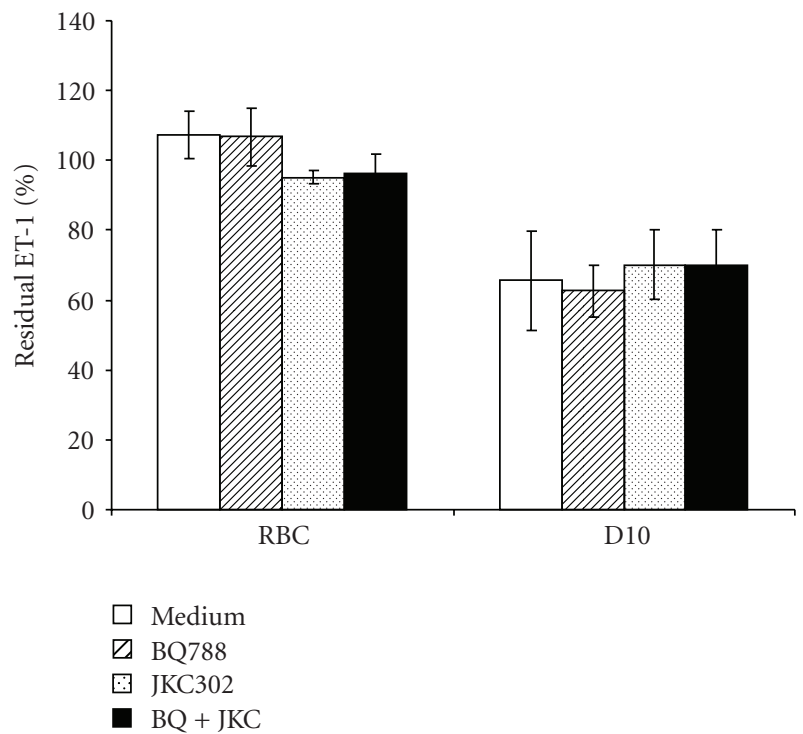

FIgURE 3: Failure of ET-1 receptor antagonists to inhibit ET-1 removal by pRBC ET-1 ( $5 \mathrm{fmol} / \mathrm{mL})$ was incubated for 24 hours with normal RBC or pRBC (D10) in medium only (empty bars) or in the presence of JKC302, an ET-A receptor antagonist (striped bars) or BQ788, an ET-B receptor antagonist (dotted bars), or in the presence of JKC302 and BQ788 simultaneously. Data are expressed as \% of ET- 1 recovered in the supernatants and represent the mean $\pm \mathrm{SD}$ of three different experiments.

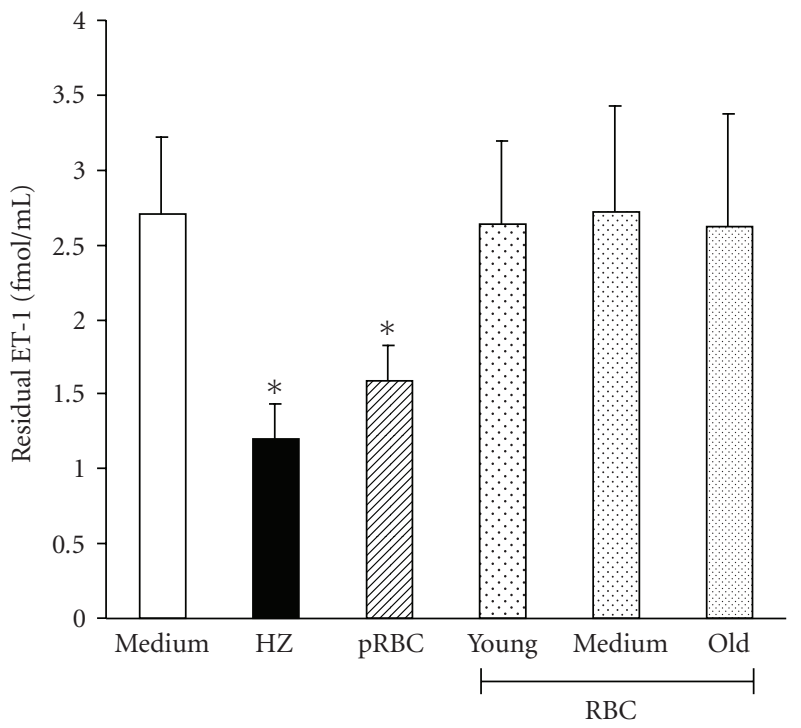

FIGURE 4: Fractions isolated by density gradient from the parasite culture have different ability to remove ET-1. Parasite culture (D10 strain) where density separated by Percoll/sorbitol gradient. Each fraction was incubated with synthetic ET-1 $(2.5 \mathrm{fmol} / \mathrm{mL})$ for 24 hours and, at end of the incubation, ET-1 was determined by ELISA. Recovery of ET-1 incubated in: medium (empty bar); RBC separated by different density and age (subdivided in young, medium and old) (dotted bars); pRBC at the trophozoite stage (4$8 \%$ parasitemia) (striped bar); fraction containing HZ (black bar). Results represent the mean \pm SD from three different experiments. $P=.007 \mathrm{HZ}$ versus control; $P=.038$ pRBC versus control. 


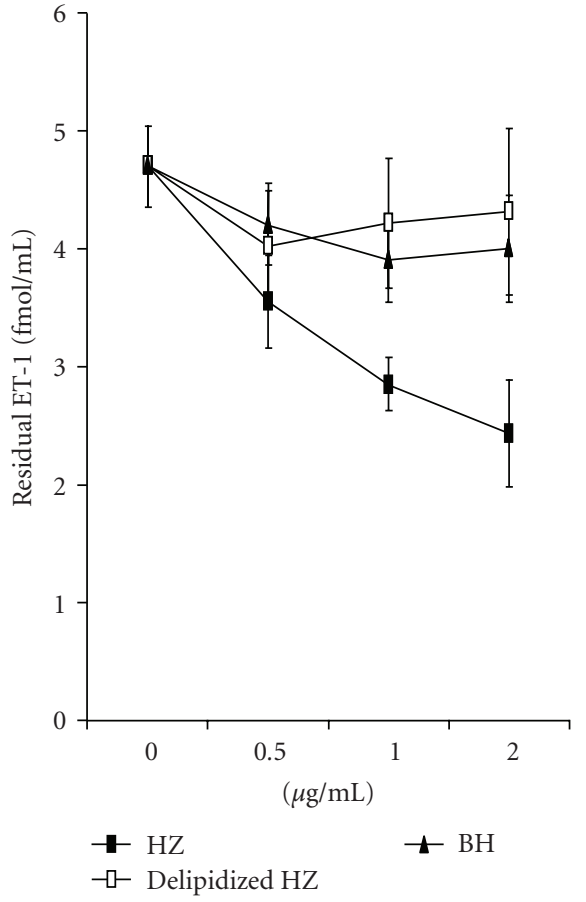

(a)

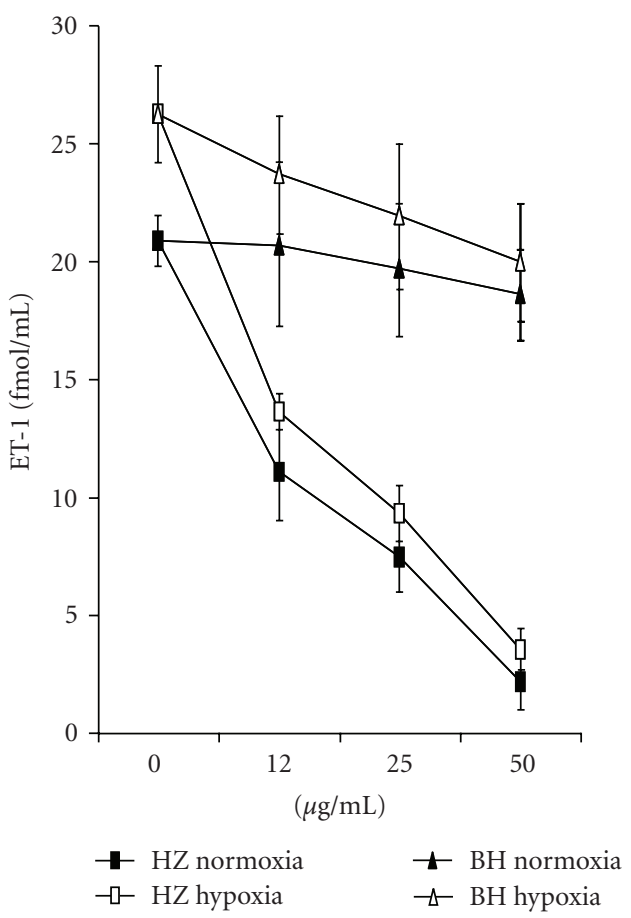

(b)

FIGURE 5: Effect of incubation of haemozoin with ET-1 synthetic or produced by HMEC-1. (a) ET-1 (5 fmol/mL) was incubated for 24 hours in the presence of different concentrations of native HZ (black square), delipidized HZ (empty square), or BH (triangle). After incubation the recovery of ET-1 was determined by ELISA. Results represent mean \pm SD from three different experiments. (b) HMECs-1 were treated for 24 hours in the presence of different concentration of native HZ (square), BH (triangle) under normoxic (black symbols), or hypoxic conditions (empty symbols). Supernatants were assayed for the presence of ET-1 by ELISA. Results represent mean \pm SD from three different experiments.

lipids; after density fractionation, only fractions enriched in pRBC and the HZ containing fraction can bind ET-1, but not normal RBCs, delipidized native HZ or synthetic BH. ET-1 can be adsorbed by neutral phospholipids via electrostatic interactions [30].

The process of $\mathrm{HZ}$ formation and the modified lipid composition of the membrane following malaria infection $[28,35]$ may explain why ET-1 does not bind to delipidized $\mathrm{HZ}$ or synthetic $\mathrm{BH}$ or normal RBCs.

Parasite neutral lipids have recently attracted considerable attention as the intracellular environment where crucial events of the parasite metabolism occur. The detoxification of haemoglobin-derived haem into $\mathrm{HZ}$ crystals seems to take place in neutral lipid nanospheres within the parasite food vacuole [36] and different neutral lipids associated with HZ have been identified by ESI-MS/MS. The most abundant are monostearic and monopalmitic glycerol, with a small amount of polar lipids such as phosphatidylcholine or phosphatidylethanolamine. These neutral lipids have been shown to promote $\mathrm{HZ}$ formation by increasing the solubility of haem monomers $[37,38]$. The observation that ET-1 adsorbs to the native $\mathrm{HZ}$, but not to the delipidated $\mathrm{HZ}$ or to $\mathrm{BH}$, strongly argues in favour of the absorption of ET-1 to the HZ-associated neutral lipids. Likewise, it can be speculated that ET-1 binds aspecifically to the lipids of the $\mathrm{pRBC}$ membrane. The lipid composition of $\mathrm{pRBC}$ membrane, in particular the phospholipid distribution and fatty acid pattern, is significantly modified by $P$. falciparum [28]. In particular, higher levels of the neutral sphingomyelin and lower levels of the acidic phosphatidylserine, phosphatidylinositol, and phosphatidylethanolamine compared to normal RBC of the same age/density have been described. $[28,36]$. This phospholipid pattern accounts for the higher content of palmitic and stearic acid, two saturated fatty acids shown to be associated with native $\mathrm{HZ}$ [36]. Based on these findings we can speculate that the aspecific binding of ET-1 to pRBC could be mainly related to the increased fraction of stearic and palmitic acid due to the different phospholipid pattern of pRBC. Another parasite product, the glycosylphosphatidylinositol (GPI), that is known to modulate endothelial cells functions, would be a candidate for further ET-1 binding studies [39].

The conditions used in these experiments are biologically sound. pRBC concentrations were $2-4 \%$, and hyperparasitaemia in falciparum malaria is defined as $\geq 4 \%$ pRBCs in the peripheral blood (locally in the small vessels of the brain and other target organs it may be much higher). The $\mathrm{HZ}$ concentration of $2 \mu \mathrm{g} / \mathrm{mL}$ is also relevant to biological 
condition; in fact it has been calculated that $\sim 3.9$ million trophozoites contain $47 \mathrm{fg}$ of haemozoin per cell with parasite at 32 hours in the life cycle [32]. Considering a $42 \%$ haematocrit, a $0.1 \%$ parasitaemia would be sufficient to release $2 \mu \mathrm{g} / \mathrm{mL}$ of haemozoin in vivo.

The clinical findings whereby ET-1 levels are unrelated to parasitaemia or severity of malaria [23] are not contradictory but simply hardly relevant to the events occurring in the small vessels of the target organs (brain, lungs, placenta). ET1 action is mainly local [14]. In these vessels, the jamming of infected and uninfected RBCs will cause hypoxia which triggers the release of ET-1 by endothelial cells. However, accumulating pRBCs will increasingly be removing ET- 1 and preventing it from exerting its vasoconstrictive action. The consequent vasodilation may ameliorate locally the blood flow but may also contribute to perivascular oedema that plays a crucial role in the pathogenesis of paediatric severe malaria and in fatal cases in adults $[40,41]$. Cerebral oedema is a common finding with modern techniques such as the magnetic resonance imaging (MRI) (T. Taylor, personal communication). In conclusion, we have shown that ET-1, but not its precursor, big ET-1, is efficiently bound in vitro by $\mathrm{pRBC}$ or $\mathrm{HZ}$ through the lipids contained within the $\mathrm{HZ}$ crystals or present in the membrane of pRBC. In vivo, in the local microenvironment where $\mathrm{pRBC}$ sequester, high levels of ET-1 are likely to be produced, particularly in cerebral malaria or in the placenta of pregnant women with malaria. Binding to $\mathrm{pRBC}$ or HZ may neutralize ET-1 activity leading to reduced vasoconstriction and reduced inflammation. The extent of the decrease in ET-1 bioavailability will be different in different areas as function of parasite density and/or HZ release. As feed back effect, the endothelial cells will augment the production of nitric oxide $(\mathrm{NO})$ which is reported to have beneficial effects in murine cerebral malaria [42].The interaction between host, parasites, and treatment are complex. The mortality caused by severe falciparum malaria remains $\sim 10-25 \%$ in spite of effective antimalarial treatment [43]. Reducing parasitaemia alone may not be enough to stop or reverse the pathological processes associated with malaria complications. This means that, even with reduced parasitaemia with antimalarials, adjunctive treatments need to be investigated to stop the progression of disease.

\section{Acknowledgments}

The authors thank Professor Timothy J Egan for helpful scientific discussion. This publication was generated in the context of the AntiMal project, funded under the 6th Framework Programme of the European Community (Contract $\mathrm{N}^{\circ}$ IP-018834 to DT). The authors are solely responsible for its content; it does not represent the opinion of the European Community and the Community is not responsible for any use that might be made of the information contained therein. Likewise, the views expressed in this publication do not necessarily represent the decisions, policy, or views of the WHO. The financial support of the Università di Milano (First 2006-2008) is also acknowledged.

\section{References}

[1] J. G. Breman and C. N. Holloway, "Malaria surveillance counts," The American Journal of Tropical Medicine and Hygiene, vol. 77, no. 6, supplement, pp. 36-47, 2007.

[2] B. C. Urban, T. T. Hien, N. P. Day, et al., "Fatal Plasmodium falciparum malaria causes specific patterns of splenic architectural disorganization," Infection and Immunity, vol. 73, no. 4, pp. 1986-1994, 2005.

[3] A. M. Dondorp, E. Pongponratn, and N. J. White, "Reduced microcirculatory flow in severe falciparum malaria: pathophysiology and electron-microscopic pathology," Acta Tropica, vol. 89, no. 3, pp. 309-317, 2004.

[4] L. Schofield and G. E. Grau, "Immunological processes in malaria pathogenesis," Nature Reviews Immunology, vol. 5, no. 9, pp. 722-735, 2005.

[5] I. A. Clark, L. M. Alleva, A. C. Mills, and W. B. Cowden, "Pathogenesis of malaria and clinically similar conditions," Clinical Microbiology Reviews, vol. 17, no. 3, pp. 509-539, 2004.

[6] D. Taramelli, N. Basilico, A. M. De Palma, et al., "The effect of synthetic malaria pigment ( $\beta$-haematin) on adhesion molecule expression and interleukin- 6 production by human endothelial cells," Transactions of the Royal Society of Tropical Medicine and Hygiene, vol. 92, no. 1, pp. 57-62, 1998.

[7] D. Taramelli, S. Recalcati, N. Basilico, P. Olliaro, and G. Cairo, "Macrophage preconditioning with synthetic malaria pigment reduces cytokine production via heme iron-dependent oxidative stress," Laboratory Investigation, vol. 80, no. 12, pp. 1781$1788,2000$.

[8] N. Basilico, C. Tognazioli, S. Picot, F. Ravagnani, and D. Taramelli, "Synergistic and antagonistic interactions between haemozoin and bacterial endotoxin on human and mouse macrophages," Parassitologia, vol. 45, no. 3-4, pp. 135-140, 2003.

[9] P. Parroche, F. N. Lauw, N. Goutagny, et al., "Malaria hemozoin is immunologically inert but radically enhances innate responses by presenting malaria DNA to Toll-like receptor 9," Proceedings of the National Academy of Sciences of the United States of America, vol. 104, no. 6, pp. 1919-1924, 2007.

[10] F. Omodeo-Salè, N. Basilico, M. Folini, P. Olliaro, and D. Taramelli, "Macrophage populations of different origins have distinct susceptibilities to lipid peroxidation induced by $\beta$ haematin (malaria pigment)," FEBS Letters, vol. 433, no. 3, pp. 215-218, 1998.

[11] D. Taramelli, "The heme moiety of malaria pigment $(\beta-$ Hematin) mediates the inhibition of nitric oxide and tumor necrosis factor- $\alpha$ production by lipopolysaccharidestimulated macrophages," Experimental Parasitology, vol. 81, no. 4, pp. 501-511, 1995.

[12] E. Schwarzer, O. A. Skorokhod, V. Barrera, and P. Arese, "Hemozoin and the human monocyte-a brief review of their interactions," Parassitologia, vol. 50, no. 1-2, pp. 143-145, 2008.

[13] Y. Sato, J. C. Hogg, D. English, and S. F. Van Eeden, "Endothelin-1 changes polymorphonuclear leukocytes' deformability and CD11b expression and promotes their retention in the lung," American Journal of Respiratory Cell and Molecular Biology, vol. 23, no. 3, pp. 404-410, 2000.

[14] R. M. Kedzierski and M. Yanagisawa, "Endothelin system: the double-edged sword in health and disease," Annual Review of Pharmacology and Toxicology, vol. 41, pp. 851-876, 2001. 
[15] T. Masaki, "Historical review: endothelin," Trends in Pharmacological Sciences, vol. 25, no. 4, pp. 219-224, 2004.

[16] C.-L. Lin, A. Y. Jeng, S.-L. Howng, and A.-L. Kwan, "Endothelin and subarachnoid hemorrhage-induced cerebral vasospasm: pathogenesis and treatment," Current Medicinal Chemistry, vol. 11, no. 13, pp. 1779-1791, 2004.

[17] W. Neuhofer and D. Pittrow, "Role of endothelin and endothelin receptor antagonists in renal disease," European Journal of Clinical Investigation, vol. 36, supplement 3, pp. 78-88, 2006.

[18] G. Callera, R. Tostes, C. Savoia, M. N. Muscara, and R. M. Touyz, "Vasoactive peptides in cardiovascular (patho)physiology," Expert Review of Cardiovascular Therapy, vol. 5, no. 3, pp. 531-552, 2007.

[19] M. Wittner, G. J. Christ, H. Huang, et al., "Trypanosoma cruzi induces endothelin release from endothelial cells," Journal of Infectious Diseases, vol. 171, no. 2, pp. 493-497, 1995.

[20] J. R. Wahl, N. J. Goetsch, H. J. Young, et al., "Murine macrophages produce endothelin-1 after microbial stimulation," Experimental Biology and Medicine, vol. 230, no. 9, pp. 652-658, 2005.

[21] M. Wanecek, E. Weitzberg, A. Rudehill, and A. Oldner, "The endothelin system in septic and endotoxin shock," European Journal of Pharmacology, vol. 407, no. 1-2, pp. 1-15, 2000.

[22] C. Wenisch, H. Wenisch, P. Wilairatana, et al., "Big endothelin in patients with complicated Plasmodium falciparum malaria," Journal of Infectious Diseases, vol. 173, no. 5, pp. 1281-1284, 1996.

[23] A. Dietmann, P. Lackner, R. Helbok, et al., "Opposed circulating plasma levels of endothelin-1 and C-type natriuretic peptide in children with Plasmodium falciparum malaria," Malaria Journal, vol. 7, no. 1, p. 253, 2008.

[24] N. Basilico, L. Speciale, S. Parapini, P. Ferrante, and D. Taramelli, "Endothelin-1 production by a microvascular endothelial cell line treated with Plasmodium falciparum parasitized red blood cells," Clinical Science, vol. 103, supplement 48, pp. 464S-466S, 2002.

[25] N. Basilico, M. Mondani, S. Parapini, L. Speciale, P. Ferrante, and D. Taramelli, "Plasmodium falciparum parasitized red blood cells modulate the production of endothelin-1 by human endothelial cells," Minerva Medica, vol. 95, no. 2, pp. 153-158, 2004.

[26] E. W. Ades, F. J. Candal, R. A. Swerlick, et al., "HMEC1: establishment of an immortalized human microvascular endothelial cell line," Journal of Investigative Dermatology, vol. 99, no. 6, pp. 683-690, 1992.

[27] W. Trager and J. B. Jensen, "Human malaria parasites in continuous culture," Science, vol. 193, no. 4254, pp. 673-675, 1976.

[28] F. Omodeo-Salè, A. Motti, N. Basilico, S. Parapini, P. Olliaro, and D. Taramelli, "Accelerated senescence of human erythrocytes cultured with Plasmodium falciparum," Blood, vol. 102, no. 2, pp. 705-711, 2003.

[29] A. Bonifacio, S. Finaurini, C. Krafft, S. Parapini, D. Taramelli, and V. Sergo, "Spatial distribution of heme species in erythrocytes infected with Plasmodium falciparum by use of resonance Raman imaging and multivariate analysis," Analytical and Bioanalytical Chemistry, vol. 392, no. 7-8, pp. 1277-1282, 2008.

[30] L. Chaloin, N. Van Mau, F. Heitz, L. Chiche, and J.-C. Talbot, "The adsorption of endothelin 1 to phospholipids is governed by electrostatic interactions: a monolayer and fluorescence study," Colloids and Surfaces B, vol. 10, no. 6, pp. 405-412, 1998.
[31] J. O. Ashong, I. P. Blench, and D. C. Warhurst, "The composition of haemozoin from Plasmodium falciparum," Transactions of the Royal Society of Tropical Medicine and Hygiene, vol. 83, no. 2, pp. 167-172, 1989.

[32] T. J. Egan, J. M. Combrinck, J. Egan, et al., "Fate of haem iron in the malaria parasite Plasmodium falciparum," Biochemical Journal, vol. 365, no. 2, pp. 343-347, 2002.

[33] A. Rivera, M. A. Rotter, and C. Brugnara, "Endothelins activate $\mathrm{Ca}^{2+}$-gated $\mathrm{K}^{+}$channels via endothelin $\mathrm{B}$ receptors in CD-1 mouse erythrocytes," American Journal of Physiology, vol. 277, no. 4, part 1, pp. C746-C754, 1999.

[34] A. Rivera, P. Jarolim, and C. Brugnara, "Modulation of Gardos channel activity by cytokines in sickle erythrocytes," Blood, vol. 99, no. 1, pp. 357-363, 2002.

[35] L. L. Hsiao, R. J. Howard, M. Aikawa, and T. F. Taraschi, "Modification of host cell membrane lipid composition by the intra-erythrocytic human malaria parasite Plasmodium falciparum," Biochemical Journal, vol. 274, no. 1, pp. 121-132, 1991.

[36] J. M. Pisciotta, I. Coppens, A. K. Tripathi, et al., "The role of eutral lipid nanospheres in Plasmodium falciparum haem crystallization," Biochemical Journal, vol. 402, no. 1, pp. 197204, 2007.

[37] T. J. Egan, "Haemozoin formation," Molecular and Biochemical Parasitology, vol. 157, no. 2, pp. 127-136, 2008.

[38] J. M. Pisciotta and D. Sullivan, "Hemozoin: oil versus water," Parasitology International, vol. 57, no. 2, pp. 89-96, 2008.

[39] L. Schofield, S. Novakovic, P. Gerold, R. T. Schwarz, M. J. McConville, and S. D. Tachado, "Glycosylphosphatidylinositol toxin of Plasmodium up-regulates intercellular adhesion molecule-1, vascular cell adhesion molecule-1, and E-selectin expression in vascular endothelial cells and increases leukocyte and parasite cytoadherence via tyrosine kinase-dependent signal transduction," Journal of Immunology, vol. 156, no. 5, pp. 1886-1896, 1996.

[40] S. Looareesuwan, P. Wilairatana, S. Krishna, et al., "Magnetic resonance imaging of the brain in patients with cerebral malaria," Clinical Infectious Diseases, vol. 21, no. 2, pp. 300 309, 1995.

[41] S. Adams, H. Brown, and G. Turner, "Breaking down the blood-brain barrier: signaling a path to cerebral malaria?" Trends in Parasitology, vol. 18, no. 8, pp. 360-366, 2002.

[42] I. Gramaglia, P. Sobolewski, D. Meays, et al., "Low nitric oxide bioavailability contributes to the genesis of experimental cerebral malaria," Nature Medicine, vol. 12, no. 12, pp. 14171422, 2006.

[43] P. Olliaro, "Mortality associated with severe Plasmodium falciparum malaria increases with age," Clinical Infectious Diseases, vol. 47, no. 2, pp. 158-160, 2008. 

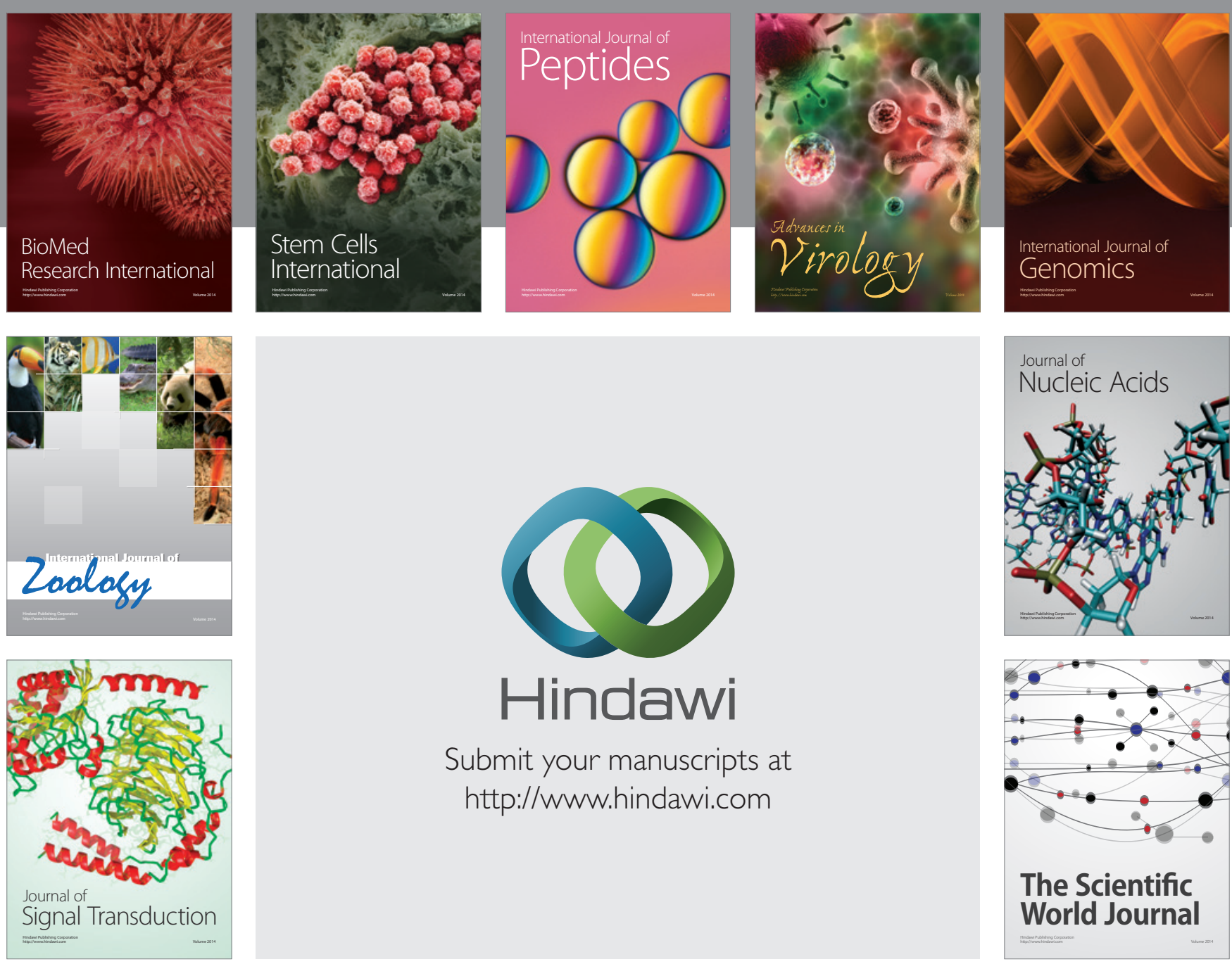

Submit your manuscripts at

http://www.hindawi.com
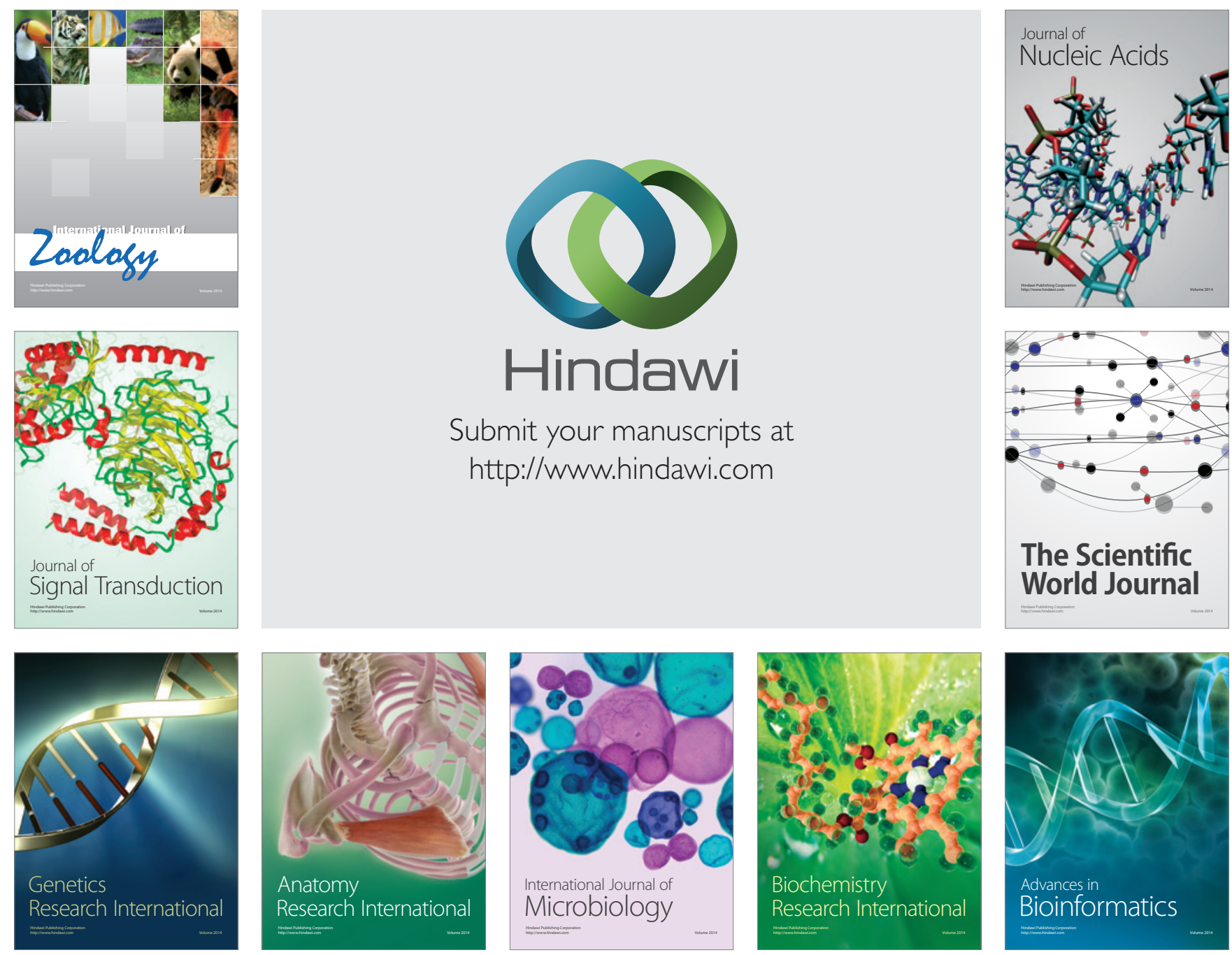

The Scientific World Journal
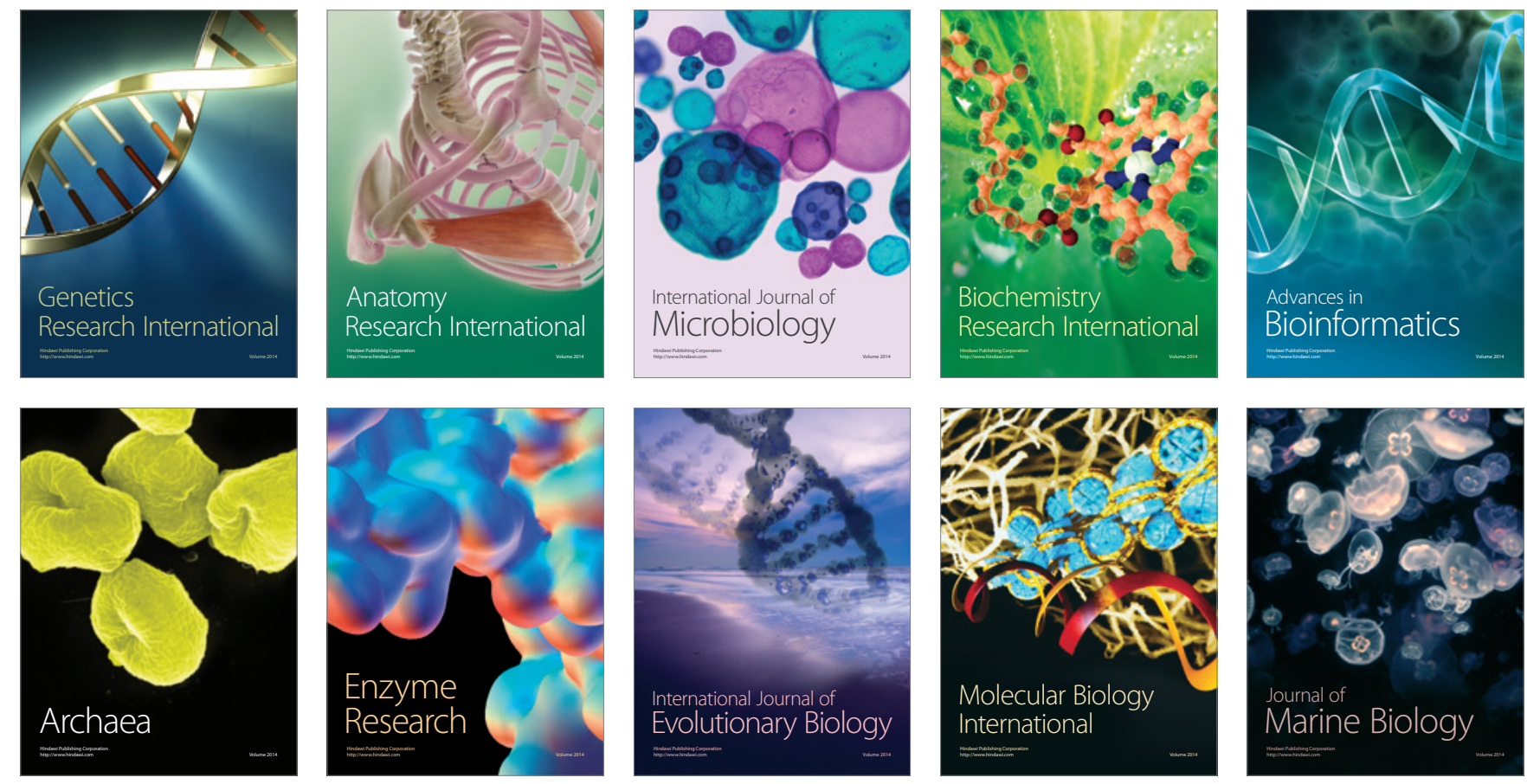